\title{
A equação de Laplace num semidisco sob a condição de fronteira mista Dirichlet-Neumann
}

\author{
Roberto Toscano Couto ${ }^{1}$ \\ UFF, Niterói, RJ
}

Resumo. Calcula-se por dois modos a solução da equação de Laplace num semidisco sob uma condição de fronteira de Dirichlet na base e uma de Neumann na circunferência. O primeiro modo consiste na superposição de uma solução calculada por separação de variáveis e uma calculada por transformada de Fourier. O segundo, no uso da função de Green determinada pelo método das imagens. Os dois modos produzem expressões idênticas para a solução.

Palavras-chave. equação de Laplace, semidisco, Dirichlet, Neumann, função de Green, método das imagens

\section{Introdução}

Neste trabalho calculamos a solução do seguinte problema:

$$
\left\{\begin{array}{l}
\nabla^{2} u(r, \theta)=\frac{\partial^{2} u}{\partial r^{2}}+\frac{1}{r} \frac{\partial u}{\partial \theta}+\frac{1}{r^{2}} \frac{\partial^{2} u}{\partial \theta^{2}}=0 \text { se } r \in(0, b) \text { e } \theta \in(0, \pi) \\
u(r, 0)=0 \text { se } r \in[0, b] \\
u(r, \pi)=f(r) \text { se } r \in(0, b] \\
\frac{\partial u}{\partial r}(b, \theta)=g(\theta) \text { se } \theta \in(0, \pi)
\end{array}\right.
$$

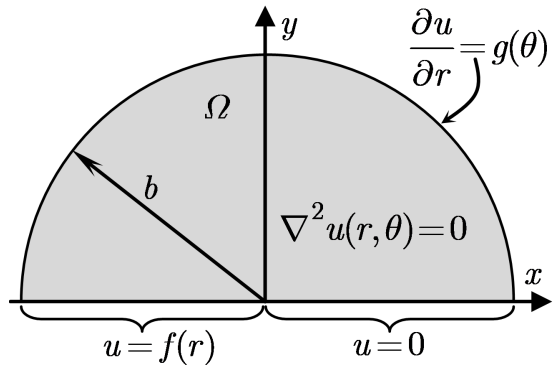

Figura 1: O problema resolvido.

A equação diferencial parcial (EDP) cuja solução se calcula é a equação de Laplace. Trata-se do problema de valor de fronteira cujo domínio $\Omega$ é o semidisco mostrado na Figura 1, onde se resolve essa EDP nas coordenadas polares sob condições de fronteira mistas, de Dirichlet na base e de Neumann na circunferência.

Dois modos de calcular a solução do problema são apresentados. Na seção 2, ela é obtida pela superposição de duas soluções, a do problema em que a condição de fronteira na base é homogeneizada (v. Figura 2), calculada por separação de variáveis, com a do problema em que tal homogeneização se dá na circunferência (v. Figura 3), calculada por transformada de Fourier. Na seção 3, a solução é calculada pela função de Green, que é determinada pelo método das imagens.

O cotejo dos dois modos de cálculo permite compreender as limitações e as vantagens de cada um. Além disso, ao fim da descrição deles, logo se percebe a possibilidade de empregá-los para resolver vários problemas derivados de modificações do problema resolvido.

\footnotetext{
${ }^{1}$ rtoscano@id.uff.br
} 


\section{Separação de variáveis e transformada de Fourier}

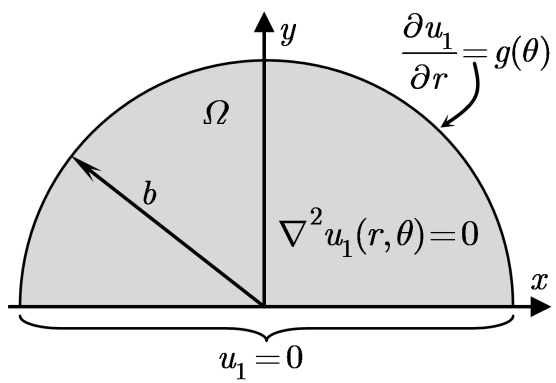

Figura 2: Problema com a condição de fronteira na base homogeneizada.

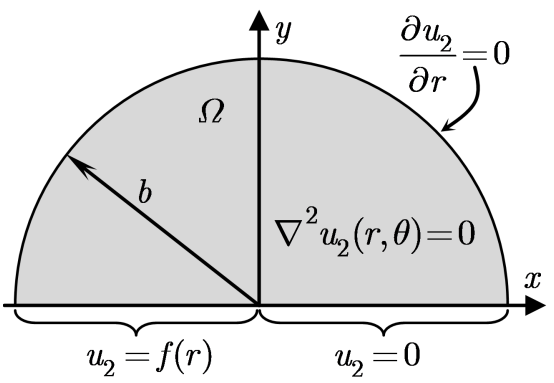

Figura 3: Problema com a condição de fronteira na circunferência homogeneizada.

A solução $u$ do problema na Figura 1 é a superposição das soluções $u_{1}$ e $u_{2}$ dos problemas nas Figuras 2 e 3, respectivamente:

$$
u(r, \theta)=u_{1}(r, \theta)+u_{2}(r, \theta) .
$$

\subsection{Resolução por separação de variáveis do problema na Figura 2}

Substituindo $u_{1}(r, \theta)=R(r) \Theta(\theta)$ em $\nabla^{2} u_{1}=0$ e separando as variáveis, obtemos

$$
R^{\prime \prime} \Theta+\frac{1}{r} R^{\prime} \Theta+\frac{1}{r^{2}} R \Theta^{\prime \prime}=0 \stackrel{\times \frac{r^{2}}{R \Theta}}{\Longrightarrow} \frac{\overbrace{r^{2} R^{\prime \prime}+r R^{\prime}}^{\lambda}}{R}+\frac{\overbrace{\Theta^{\prime \prime}}^{-\lambda}}{\Theta}=0 \Rightarrow\left\{\begin{array}{l}
\Theta^{\prime \prime}+\lambda \Theta(\theta)=0 \\
r^{2} R^{\prime \prime}+r R^{\prime}-\lambda R(r)=0
\end{array}\right.
$$

Temos que $u_{1}(r, 0)=R(r) \Theta(0)=0 \Rightarrow \Theta(0)=0$ e $u_{1}(r, \pi)=R(r) \Theta(\pi)=0 \Rightarrow \Theta(\pi)=0$; essas condições e a EDO para $\Theta(\theta)$ em (3) formam um problema de autovalor de solução bem conhecida $\{$ Ref. [5], seç. 11.4, Exemplo 1\}:

$$
\left\{\begin{array} { l } 
{ \Theta ^ { \prime \prime } + \lambda \Theta ( \theta ) = 0 , \theta \in ( 0 , \pi ) } \\
{ \Theta ( 0 ) = \Theta ( \pi ) = 0 }
\end{array} \quad \Rightarrow \quad \left\{\begin{array}{l}
\lambda_{n}=n^{2}(n=1,2,3 \cdots) \\
\Theta_{n}(\theta)=\operatorname{sen} n \theta
\end{array}\right.\right.
$$

Agora resolvemos a EDO para $R(r)$ em (3) com esses valores de $\lambda$ \{Ref. [5], seç.6.1, eq. (2)\}:

$$
r^{2} R_{n}^{\prime \prime}+r R_{n}^{\prime}-n^{2} R_{n}(r)=0 \quad \Rightarrow \quad R_{n}(r)=C_{n} r^{n}+D_{n} / r^{n},
$$

onde devemos fazer $D_{n}=0$ para evitar infinitude quando $r=0$. Logo, a solução mais geral é

$$
u_{1}(r, \theta)=\sum_{n=1}^{\infty} R_{n}(r) \Theta_{n}(\theta)=\sum_{n=1}^{\infty} C_{n} r^{n} \operatorname{sen} n \theta
$$

Calculamos as constantes $C_{n}$ impondo a condição na fronteira circular, o que resulta numa série de Fourier em senos cujos coeficientes as envolvem:

$$
\frac{\partial u_{1}}{\partial r}(b, \theta)=\sum_{n=1}^{\infty} n C_{n} b^{n-1} \operatorname{sen} n \theta=g(\theta) \quad \Rightarrow \quad n C_{n} b^{n-1}=\frac{2}{\pi} \int_{0}^{\pi} g(\theta) \operatorname{sen} n \theta d \theta .
$$

Substituindo essa expressão de $C_{n}$ (com a variável de integração $\theta$ trocada por $\theta^{\prime}$ ) em (4), obtemos

$$
u_{1}(r, \theta)=\sum_{n=1}^{\infty}\left[\frac{2}{\pi n b^{n-1}} \int_{0}^{\pi} g\left(\theta^{\prime}\right) \operatorname{sen} n \theta^{\prime} d \theta^{\prime}\right] r^{n} \operatorname{sen} n \theta=\frac{b}{\pi} \int_{0}^{\pi}[\underbrace{\sum_{n=1}^{\infty} \frac{r^{n} 2 \operatorname{sen} n \theta^{\prime} \operatorname{sen} n \theta}{n b^{n}}}_{S}] g\left(\theta^{\prime}\right) d \theta^{\prime} .
$$


O somatório $S$, usando $2 \operatorname{sen} n \theta^{\prime} \operatorname{sen} n \theta=\cos n\left(\theta^{\prime}-\theta\right)-\cos n\left(\theta^{\prime}+\theta\right)$, pode ser escrito na forma

$$
S=\sum_{n=1}^{\infty} \frac{r^{n}\left[\cos n\left(\theta^{\prime}-\theta\right)-\cos n\left(\theta^{\prime}+\theta\right)\right]}{n b^{n}}=\sigma\left(\theta^{\prime}-\theta\right)+\sigma\left(\theta^{\prime}+\theta\right) ; \quad \sigma(\varphi) \equiv \sum_{n=1}^{\infty} \frac{1}{n}\left(\frac{r}{b}\right)^{n} \cos n \varphi .
$$

Se $z \equiv(r / b) e^{\mathrm{i} \varphi}$, então $z^{n}=(r / b)^{n} e^{\mathrm{i} n \varphi}=(r / b)^{n} \cos n \varphi+\mathrm{i}(r / b)^{n} \operatorname{sen} n \varphi$, e, portanto,

$$
\begin{aligned}
\sigma(\varphi) & =\sum_{n=1}^{\infty} \frac{\operatorname{Re} z^{n}}{n}=\operatorname{Re} \sum_{n=1}^{\infty} \frac{z^{n}}{n}=\operatorname{Re} \sum_{n=1}^{\infty} \int_{0}^{z} \zeta^{n-1} d \zeta=\operatorname{Re} \int_{0}^{z}\left(\sum_{n=1}^{\infty} \zeta^{n-1}\right) d \zeta=\operatorname{Re} \int_{0}^{z}\left(\sum_{n=0}^{\infty} \zeta^{n}\right) d \zeta \\
& =\operatorname{Re} \int_{0}^{z} \frac{d \zeta}{1-\zeta}=\operatorname{Re}[-\ln (1-z)]=-\operatorname{Re}[\ln |1-z|+\mathrm{i} \arg (1-z)]=-\ln |1-z|=-\ln \left|1-\frac{r}{b} e^{\mathrm{i} \varphi}\right| \\
& =-\ln \left|1-\frac{r}{b} \cos \varphi-\mathrm{i} \frac{r}{b} \operatorname{sen} \varphi\right|=-\ln \sqrt{\left(1-\frac{r}{b} \cos \varphi\right)^{2}+\left(\frac{r}{b} \operatorname{sen} \varphi\right)^{2}} \\
& =-\frac{1}{2} \ln \left(1-\frac{2 r}{b} \cos \varphi+\frac{r^{2}}{b^{2}}\right)=-\frac{1}{2} \ln \frac{b^{2}-2 b r \cos \varphi+r^{2}}{b^{2}}=-\frac{1}{2} \ln \left[b^{2}-2 b r \cos \varphi+r^{2}\right]+\ln b .
\end{aligned}
$$

Esse resultado, levado em (6), produz

$$
S=-\frac{1}{2} \ln \left[b^{2}-2 b r \cos \left(\theta^{\prime}-\theta\right)+r^{2}\right]+\frac{1}{2} \ln \left[b^{2}-2 b r \cos \left(\theta^{\prime}+\theta\right)+r^{2}\right]=\frac{1}{2} \ln \frac{b^{2}-2 b r \cos \left(\theta^{\prime}+\theta\right)+r^{2}}{b^{2}-2 b r \cos \left(\theta^{\prime}-\theta\right)+r^{2}},
$$

o qual, por sua vez, levado em (5), fornece, finalmente,

$$
u_{1}(r, \theta)=\frac{b}{2 \pi} \int_{0}^{\pi} \ln \frac{b^{2}-2 b r \cos \left(\theta^{\prime}+\theta\right)+r^{2}}{b^{2}-2 b r \cos \left(\theta^{\prime}-\theta\right)+r^{2}} g\left(\theta^{\prime}\right) d \theta^{\prime} .
$$

\subsection{Resolução por transformada de Fourier do problema na Figura 3}

Se mudarmos para a variável

$$
\rho=-\ln (r / b) \quad\left[\Rightarrow \quad r=b e^{-\rho}, \quad \operatorname{com} \rho \in(0, \infty)\right],
$$

a $\operatorname{EDP} \nabla^{2} u_{2}(r, \theta)=0$, ou $r^{2} \partial^{2} u_{2} / \partial r^{2}+r \partial u_{2} / \partial r+\partial^{2} u_{2} / \partial \theta^{2}=0$, toma a forma

$$
\partial^{2} U_{2} / \partial \rho^{2}+\partial^{2} U_{2} / \partial \theta^{2}=0 \quad\left[u_{2}(r, \theta)=u_{2}\left(b e^{-\rho}, \theta\right) \equiv U_{2}(\rho, \theta)\right],
$$

pois, pela regra da cadeia, conclui-se que

$$
\text { (i) } r \frac{\partial u_{2}}{\partial r}(r, \theta)=-\frac{\partial U_{2}}{\partial \rho}(\rho, \theta) \quad \text { e } \quad \text { (ii) } r^{2} \frac{\partial^{2} u_{2}}{\partial r^{2}}=\frac{\partial^{2} U_{2}}{\partial \rho^{2}}+\frac{\partial U_{2}}{\partial \rho} \text {. }
$$

Aplicando em (10) a transformada de Fourier em cossenos definida por

$$
\mathcal{F}_{c}\left\{U_{2}(\rho, \theta)\right\}=\sqrt{\frac{2}{\pi}} \int_{0}^{\infty} U_{2}(\rho, \theta) \cos k \rho d \rho \equiv \bar{U}_{2}(k, \theta)
$$

obtemos \{Ref. [1], seç.7.6, p.279\}

$$
\begin{aligned}
& -k^{2} \bar{U}_{2}(k, \theta)-\sqrt{\frac{2}{\pi}} \overbrace{\left.\frac{\partial U_{2}}{\partial \rho}(\rho, \theta)\right|_{\rho=0}}^{0}+\frac{d^{2} \bar{U}_{2}}{d \theta^{2}}(k, \theta)=0 \Rightarrow \frac{d^{2} \bar{U}_{2}}{d \theta^{2}}-k^{2} \bar{U}_{2}(k, \theta)=0 \\
& \Rightarrow \bar{U}_{2}(k, \theta)=c_{1} \cosh k \theta+c_{2} \operatorname{senh} k \theta,
\end{aligned}
$$

onde o termo indicado como nulo é explicado com base na equação (i) em (11) e na condição homogênea na circunferência: $\left.\frac{\partial U_{2}}{\partial \rho}(\rho, \theta)\right|_{\rho=0}=-r \underbrace{\left.\frac{\partial u_{2}}{\partial r}(r, \theta)\right|_{r=b}}_{0}=0$.

Para calcular as constantes $c_{1}$ e $c_{2}$ em (13), usamos as condições de Dirichlet na base do semidisco, sendo necessário, antes, tomar a transformada de Fourier em cossenos delas: 
$u_{2}(r, 0)=0 \Longrightarrow U_{2}(\rho, 0)=0 \stackrel{\mathcal{F}_{c}}{\Longrightarrow} \quad \bar{U}_{2}(k, 0)=c_{1}=0$.
$u_{2}(r, \pi)=f(r) \Rightarrow U_{2}(\rho, \pi)=F(\rho) \stackrel{\mathcal{F}_{c}}{\Longrightarrow} \bar{U}_{2}(k, \pi)=c_{2} \operatorname{senh} k \pi=\mathcal{F}_{c}\{F(\rho)\} \Rightarrow c_{2}=\mathcal{F}_{c}\{F(\rho)\} / \operatorname{senh} k \pi$, onde definimos $f(r)=f\left(b e^{-\rho}\right) \equiv F(\rho)$. Com $c_{1}$ e $c_{2}$ assim determinados, (13) passa a ser

$$
\bar{U}_{2}(k, \theta)=\frac{\mathcal{F}_{c}\{F(\rho)\}}{\operatorname{senh} k \pi} \operatorname{senh} k \theta=\sqrt{\frac{2}{\pi}} \frac{\operatorname{senh} k \theta}{\operatorname{senh} k \pi} \int_{0}^{\infty} F\left(\rho^{\prime}\right) \cos k \rho^{\prime} d \rho^{\prime} .
$$

Tomando a transformada inversa e trocando a ordem das integrações, obtemos

$$
\begin{aligned}
U_{2}(\rho, \theta) & =\mathcal{F}_{c}^{-1}\left\{\bar{U}_{2}(k, \theta)\right\}=\mathcal{F}_{c}^{-1}\left\{\sqrt{\frac{2}{\pi}} \frac{\operatorname{senh} k \theta}{\operatorname{senh} k \pi} \int_{0}^{\infty} F\left(\rho^{\prime}\right) \cos k \rho^{\prime} d \rho^{\prime}\right\} \\
& =\sqrt{\frac{2}{\pi}} \int_{0}^{\infty}\left\{\sqrt{\frac{2}{\pi}} \frac{\operatorname{senh} k \theta}{\operatorname{senh} k \pi} \int_{0}^{\infty} F\left(\rho^{\prime}\right) \cos k \rho^{\prime} d \rho^{\prime}\right\} \cos k \rho d k \\
& =\frac{1}{\pi} \int_{0}^{\infty}(\underbrace{\int_{0}^{\infty} \frac{\operatorname{senh} k \theta}{\operatorname{senh} k \pi} 2 \cos k \rho^{\prime} \cos k \rho d k}_{I}) F\left(\rho^{\prime}\right) d \rho^{\prime} .
\end{aligned}
$$

Para calcular a integral denotada acima por $I$, usamos a identidade trigonométrica $2 \cos k \rho^{\prime} \cos k \rho$ $=\cos k\left(\rho^{\prime}-\rho\right)+\cos k\left(\rho^{\prime}+\rho\right)$, em seguida (na passagem indicada por $\stackrel{*}{=}$ ) valemo-nos da fórmula 3.981-5 listada na referência [3], e então eliminamos $\rho$ e $\rho^{\prime}$, voltando para a variável $r$ e $r^{\prime}$, obtendo

$$
\begin{aligned}
I & =\int_{0}^{\infty} \frac{\operatorname{senh} k \theta}{\operatorname{senh} k \pi} 2 \cos k \rho^{\prime} \cos k \rho d k=\int_{0}^{\infty} \frac{\operatorname{senh} k \theta}{\operatorname{senh} k \pi} \cos k\left(\rho^{\prime}-\rho\right) d k+\int_{0}^{\infty} \frac{\operatorname{senh} k \theta}{\operatorname{senh} k \pi} \cos k\left(\rho^{\prime}+\rho\right) d k \\
& \stackrel{*}{=} \frac{(1 / 2) \operatorname{sen} \theta}{\cosh \left(\rho^{\prime}-\rho\right)+\cos \theta}+\frac{(1 / 2) \operatorname{sen} \theta}{\cosh \left(\rho^{\prime}+\rho\right)+\cos \theta}=\frac{(1 / 2) \operatorname{sen} \theta}{\frac{r^{\prime 2}+r^{2}}{2 r r^{\prime}}+\cos \theta}+\frac{(1 / 2) \operatorname{sen} \theta}{\frac{b^{2}+\left(r r^{\prime} / b\right)^{2}}{2 r r^{\prime}}+\cos \theta} \\
& =\left[\frac{1}{r^{\prime 2}+r^{2}+2 r r^{\prime} \cos \theta}+\frac{1}{\left(r r^{\prime} / b\right)^{2}+b^{2}+2 r r^{\prime} \cos \theta}\right] r r^{\prime} \operatorname{sen} \theta .
\end{aligned}
$$

Substituindo esse resultado em (14), tendo em conta que $\int_{0}^{\infty} F\left(\rho^{\prime}\right) d \rho^{\prime}=\int_{b}^{0} f\left(r^{\prime}\right)\left(-d r^{\prime} / r^{\prime}\right)$, e lembrando que $U_{2}(\rho, \theta)=u_{2}(r, \theta)$, concluímos o cálculo desta seção:

$$
u_{2}(r, \theta)=\frac{r \operatorname{sen} \theta}{\pi} \int_{0}^{b}\left[\frac{1}{r^{\prime 2}+r^{2}+2 r r^{\prime} \cos \theta}+\frac{1}{\left(r r^{\prime} / b\right)^{2}+b^{2}+2 r r^{\prime} \cos \theta}\right] f\left(r^{\prime}\right) d r^{\prime} .
$$

\section{Função de Green determinada pelo método das imagens}

\subsection{Formulação}

O valor da solução $u$ do problema em (1) num ponto $\vec{r}$ do domínio $\Omega$ pode ser expresso pela seguinte integral de linha na fronteira $\partial \Omega$ (percorrida na orientação positiva convencional), conhecida como representação de Green \{Ref. [4], seç. VII.9; Ref. [2], eq. (5.0.13)\}:

$$
u(\vec{r})=\frac{1}{2 \pi} \int_{\partial \Omega}\left[G\left(\vec{r} \mid \vec{r}^{\prime}\right) \frac{\partial u}{\partial n^{\prime}}\left(\vec{r}^{\prime}\right)-\frac{\partial G}{\partial n^{\prime}}\left(\vec{r} \mid \vec{r}^{\prime}\right) u\left(\vec{r}^{\prime}\right)\right] d s^{\prime},
$$

sendo, nesse caso bidimensional, a função de Green $G\left(\vec{r} \mid \vec{r}^{\prime}\right)$ dada pela soma da função $\mathcal{H}\left(\left|\vec{r}^{\prime}-\vec{r}\right|\right) \equiv$ $\ln \left[1 /\left|\vec{r}^{\prime}-\vec{r}\right|\right]$, harmônica se $\vec{r}^{\prime} \neq \vec{r}\left\{\right.$ Ref. [4], Exemplo VII.3.1\}, com qualquer outra função $v\left(\vec{r}^{\prime}\right)$ harmônica em $\Omega$, isto é,

$$
\left\{\begin{array}{l}
G\left(\vec{r} \mid \vec{r}^{\prime}\right)=\ln \frac{1}{\left|\vec{r}^{\prime}-\vec{r}\right|}+v\left(\vec{r}^{\prime}\right), \text { com } \vec{r} \text { e } \vec{r}^{\prime} \text { em } \bar{\Omega} \equiv \Omega \cup \partial \Omega \\
\text { onde } \nabla^{\prime 2} v\left(\vec{r}^{\prime}\right)=0 \text { se } \vec{r}^{\prime} \in \Omega
\end{array}\right.
$$


Seja $\partial \Omega=\partial \Omega_{1} \cup \partial \Omega_{2}$, onde $\partial \Omega_{1}$ e $\partial \Omega_{2}$ representam a base e a circunferência do semidisco, respectivamente. Com as exigências

$$
\text { (i) } G\left(\vec{r} \mid \vec{r}^{\prime}\right)=0 \text { se } \vec{r}^{\prime} \in \partial \Omega_{1} \quad \text { e } \quad \text { (ii) } \frac{\partial G}{\partial n^{\prime}}\left(\vec{r} \mid \vec{r}^{\prime}\right)=0 \text { se } \vec{r}^{\prime} \in \partial \Omega_{2}
$$

porque não se conhece $\partial u / \partial n^{\prime}$ em $\partial \Omega_{1}$ nem $u$ em $\partial \Omega_{2}$, a equação (17) passa a fornecer a solução do problema em (1):

$$
u(\vec{r})=\underbrace{-\frac{1}{2 \pi} \int_{\partial \Omega_{1}} \frac{\partial G}{\partial n^{\prime}}\left(\vec{r} \mid \vec{r}^{\prime}\right) u\left(\vec{r}^{\prime}\right) d s^{\prime}}_{u_{2}(r, \theta)}+\underbrace{\frac{1}{2 \pi} \int_{\partial \Omega_{2}} G\left(\vec{r} \mid \vec{r}^{\prime}\right) \frac{\partial u}{\partial n^{\prime}}\left(\vec{r}^{\prime}\right) d s^{\prime}}_{u_{1}(r, \theta)} .
$$

As exigências em (20) são satisfeitas determinando-se a função harmônica $v\left(\vec{r}^{\prime}\right)$ em (18) apropriada. Aqui empregamos o método das imagens para determinar $v\left(\vec{r}^{\prime}\right)$.

Na expressão acima, da solução em termos da função de Green, indicamos um dos termos por $u_{1}(r, \theta)$ e o outro por $u_{2}(r, \theta)$, pois mostramos nesta seção que eles são exatamente aqueles em (8) e (16), respectivamente, cuja soma, como (2) indica, é a solução do problema definido em (1).

\subsection{Determinação da função de Green}

Observe que a função harmônica $\mathcal{H}\left(\left|\vec{r}^{\prime}-\vec{r}\right|\right)=$ $\ln \left(1 /\left|\vec{r}^{\prime}-\vec{r}\right|\right)$ em (18) é singular se $\vec{r}^{\prime}=\vec{r}$, e, como estamos considerando $\vec{r}^{\prime}$ como a variável vetorial (as variáveis são as coordenadas desse vetor), dizemos que $\vec{r}$ é a singularidade de $\mathcal{H}\left(\left|\vec{r}^{\prime}-\vec{r}\right|\right)$. O problema em (1) admite que a função de Green seja determinada pelo método das imagens \{Ref. [4], seç. VII.13\}, isto é, que a função $v\left(\vec{r}^{\prime}\right)$ usada na definição em (18) seja formada por termos aditivos da forma $\pm \mathcal{H}\left(\lambda\left|\vec{r}^{\prime}-\vec{r}_{i}\right|\right)$ [com singularidades em pontos $\vec{r}_{i}$ : as imagens do ponto $\vec{r}$ ] como segue:

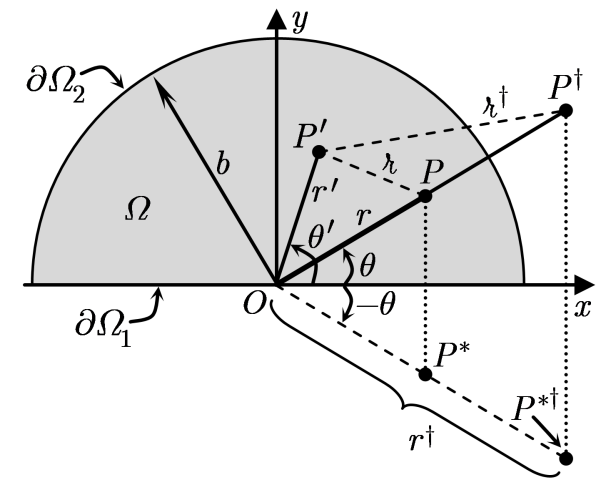

Figura 4: Configuração das imagens na determinação da função de Green.

$$
G\left(\vec{r} \mid \vec{r}^{\prime}\right)=\ln \frac{1}{\left|\vec{r}^{\prime}-\vec{r}\right|}+\overbrace{\left[-\ln \frac{1}{\left|\vec{r}^{\prime}-\vec{r}^{*}\right|}+\ln \frac{1}{\lambda\left|\vec{r}^{\prime}-\vec{r}^{\dagger}\right|}-\ln \frac{1}{\lambda\left|\vec{r}^{\prime}-\vec{r}^{* \dagger}\right|}\right]} .
$$

Para descrever os parâmetros em (22), considere a Figura (4), que, por simplificação, não mostra desenhados os vetores posição $\vec{r}^{\prime}, \vec{r}, \vec{r}^{*}, \vec{r}^{\dagger}$ e $\vec{r}^{* \dagger}$, mas apenas os respectivos pontos $P^{\prime}, P$, $P^{*}, P^{\dagger}$ e $P^{* \dagger}$ nas extremidades desses vetores (que partem da origem $O$ ). Note as coordenadas polares $r$ e $\theta$ de $P$ (ou de $\vec{r}$ ), e $r^{\prime}$ e $\theta^{\prime}$ de $P^{\prime}$ (ou de $\vec{r}^{\prime}$ ).

Neste problema, o método das imagens prescreve que as singularidades sejam posicionadas simetricamente em relação à reta contendo a base do semidisco; em outros termos, que $P^{*}$ e $P^{* \dagger}$ sejam as imagens especulares em relação ao eixo $x$ de $P$ e $P^{\dagger}$, respectivamente. Também prescreve que as imagens fora da circunferência (completa) sejam geradas pela transformação de inversão em relação à circunferência \{Ref.[4], seç. VII.3\} das singularidades no interior; por essa transformação, o vetor posição da imagem tem a direção preservada, mas a sua magnitude é aumentada pelo fator $1 / \lambda^{2}, \operatorname{com} \lambda=r / b<1$. Logo, as coordenadas polares das extremidades dos vetores em (22) são

$$
P^{\prime}\left(r^{\prime}, \theta^{\prime}\right), P(r, \theta), \quad P^{*}(r,-\theta), P^{\dagger}\left(r^{\dagger}, \theta\right), \quad P^{* \dagger}\left(r^{\dagger},-\theta\right), \quad \text { com } r^{\dagger}=r / \lambda^{2} \text { e } \lambda=r / b .
$$

Em (22) aparecem módulos de diferenças de vetores que são as distâncias entre $P^{\prime}$ e os demais pontos considerados; segue uma notação para elas e suas expressões (que podem ser obtidas pela 
lei dos cossenos) em função das coordenadas polares das variáveis vetoriais $\vec{r}$ e $\vec{r}^{\prime}$ de $G\left(\vec{r} \mid \vec{r}^{\prime}\right)$ :

$$
\left\{\begin{aligned}
r & \equiv\left|\vec{r}^{\prime}-\vec{r}\right|=\sqrt{r^{\prime 2}+r^{2}-2 r^{\prime} r \cos \left(\theta^{\prime}-\theta\right)} \\
r^{\dagger} & \equiv\left|\vec{r}^{\prime}-\vec{r}^{\dagger}\right|=\sqrt{r^{\prime 2}+r^{\dagger}-2 r^{\prime} r^{\dagger} \cos \left(\theta^{\prime}-\theta\right)}=\frac{b}{r} \sqrt{\left(r r^{\prime} / b\right)^{2}+b^{2}-2 r^{\prime} r \cos \left(\theta^{\prime}-\theta\right)} \\
r^{*} & \equiv\left|\vec{r}^{\prime}-\vec{r}^{*}\right|=\sqrt{r^{\prime 2}+r^{2}-2 r^{\prime} r \cos \left(\theta^{\prime}+\theta\right)} \\
r^{* \dagger} & \equiv\left|\vec{r}^{\prime}-\vec{r}^{* \dagger}\right|=\sqrt{r^{\prime 2}+r^{\dagger}-2 r^{\prime} r^{\dagger} \cos \left(\theta^{\prime}+\theta\right)}=\frac{b}{r} \sqrt{\left(r r^{\prime} / b\right)^{2}+b^{2}-2 r^{\prime} r \cos \left(\theta^{\prime}+\theta\right)}
\end{aligned}\right.
$$

A inversão em relação a uma circunferência se baseia \{v. Ref. [2], Fig.5.7.1\} em se exigir a validade da equação $\lambda r^{\dagger}=r$ (no caso dos pontos $P$ e $P^{\dagger}$ ) para qualquer $\vec{r}^{\prime}$ na circunferência, isto é, quando $r^{\prime}=b$. Usando $b$ como subíndice de qualquer das distâncias em (24) quando $P^{\prime}$ jaz na circunferência, essa relação básica entre $r$ e $r^{\dagger}$, e entre $r^{*}$ e $r^{* \dagger}$, pode ser escrita na forma

$$
\lambda r_{b}^{\dagger}=r_{b} \quad \text { e } \lambda r_{b}^{* \dagger}=r_{b}^{*}, \quad \text { onde } r_{b}=\left.r\right|_{r^{\prime}=b}, \quad r_{b}^{\dagger}=\left.r^{\dagger}\right|_{r^{\prime}=b} \text {, etc. }
$$

Que a função $v\left(\vec{r}^{\prime}\right)$ indicada em (22) satisfaz (19) decorre do fato de $\vec{r}^{*}, \vec{r}^{\dagger}$ e $\vec{r}^{* \dagger}$ não estarem em $\Omega$. Que (22) satisfaz a condição (i) em (20) segue do fato de $\left|\vec{r}^{\prime}-\vec{r}\right|=\left|\vec{r}^{\prime}-\vec{r}^{*}\right|$ e $\left|\vec{r}^{\prime}-\vec{r}^{\dagger}\right|=$ $\left|\vec{r}^{\prime}-\vec{r}^{* \dagger}\right|$ quando $\vec{r}^{\prime} \in \partial \Omega$, o que é mais evidente imaginando-se, na Figura 4, que $P^{\prime} \in \partial \Omega_{1}$.

Resta mostrar que (22) satisfaz a condição (ii) em (20), o que requer alguns cálculos. Usando (24) para reescrever (22) (note que o parâmetro $\lambda$ é cancelado), e usando (25), temos que

$$
\begin{aligned}
& G\left(\vec{r} \mid \vec{r}^{\prime}\right)=\underbrace{-\ln r-\ln r^{\dagger}}_{G_{1}}+\underbrace{\ln r^{*}+\ln r^{* \dagger}}_{G_{2}} . \\
& \left.\therefore \frac{\partial G_{1}}{\partial n^{\prime}}\left(\vec{r} \mid \vec{r}^{\prime}\right)\right|_{\vec{r}^{\prime} \in \partial \Omega_{2}}=\left.\frac{\partial G_{1}}{\partial r^{\prime}}\right|_{r^{\prime}=b}=\left[-\frac{1}{r} \frac{\partial r}{\partial r^{\prime}}-\frac{1}{r^{\dagger}} \frac{\partial r^{\dagger}}{\partial r^{\prime}}\right]_{r^{\prime}=b} \\
& =-\frac{1}{r_{b}} \frac{b-r \cos \left(\theta^{\prime}-\theta\right)}{r_{b}}-\underbrace{\frac{1}{r_{b}^{\dagger} r / b}}_{r_{b}} \underbrace{\frac{r^{2} / b-r \cos \left(\theta^{\prime}-\theta\right)}{\sqrt{b^{2}+r^{2}-2 b r \cos \left(\theta^{\prime}-\theta\right)}}}_{r_{b}}=-\frac{\overbrace{b^{2}+r^{2}-2 b r \cos \left(\theta^{\prime}-\theta\right)}^{b r_{b}^{2}}}{b}=-\frac{1}{b} \text {. }
\end{aligned}
$$

Analogamente obtém-se $\partial G_{2} /\left.\partial n^{\prime}\right|_{\vec{r}^{\prime} \in \partial \Omega_{2}}=1 / b$. Logo, se $\vec{r}^{\prime} \in \partial \Omega_{2}$, então $\partial G / \partial n^{\prime}=\partial G_{1} / \partial n^{\prime}+$ $\partial G_{2} / \partial n^{\prime}=-1 / b+1 / b=0$, provando (ii) em (20). Está assim comprovado que (22), ou (26), é a função de Green do problema em (1).

\section{Cálculo da solução}

Usando (26) e (24), calculemos a parcela da solução dada por (21) que é lá designada por $u_{1}(r, \theta)$. O integrando neste termo é assim desenvolvido:

$$
\begin{aligned}
& {\left[G\left(\vec{r} \mid \vec{r}^{\prime}\right) \frac{\partial u}{\partial n^{\prime}}\left(\vec{r}^{\prime}\right) d s^{\prime}\right]_{\vec{r}^{\prime} \in \partial \Omega_{2}}=\left[\ln \left(\frac{r^{*} r^{* \dagger}}{r^{\dagger}}\right) \frac{\partial u}{\partial r^{\prime}}\left(r^{\prime}, \theta^{\prime}\right) b d \theta^{\prime}\right]_{r^{\prime}=b}} \\
& =b \ln \frac{\sqrt{b^{2}+r^{2}-2 b r \cos \left(\theta^{\prime}+\theta\right)} \text { (bfr) } \sqrt{r^{2}+b^{2}-2 b r \cos \left(\theta^{\prime}+\theta\right)}}{\sqrt{b^{2}+r^{2}-2 b r \cos \left(\theta^{\prime}-\theta\right)} \text { (bfr) } \sqrt{r^{2}+b^{2}-2 b r \cos \left(\theta^{\prime}-\theta\right)}} g\left(\theta^{\prime}\right) d \theta^{\prime} \\
& =b \ln \left[\frac{r^{2}+b^{2}-2 b r \cos \left(\theta^{\prime}+\theta\right)}{r^{2}+b^{2}-2 b r \cos \left(\theta^{\prime}-\theta\right)}\right] g\left(\theta^{\prime}\right) d \theta^{\prime} .
\end{aligned}
$$

Substituindo este resultado, obtemos exatamente a equação (8), resultante da separação de variáveis:

$$
u_{1}(r, \theta)=\frac{b}{2 \pi} \int_{0}^{\pi} \ln \left[\frac{r^{2}+b^{2}-2 b r \cos \left(\theta^{\prime}+\theta\right)}{r^{2}+b^{2}-2 b r \cos \left(\theta^{\prime}-\theta\right)}\right] g\left(\theta^{\prime}\right) d \theta^{\prime} .
$$


Calculemos agora a parcela da solução dada por (21) que é lá designada por $u_{2}(r, \theta)$. Usando (26) e (24), obtemos a seguinte expressão para o integrando neste termo:

$$
\begin{aligned}
& {\left[\frac{\partial G}{\partial n^{\prime}}\left(\vec{r} \mid \vec{r}^{\prime}\right) u\left(\vec{r}^{\prime}\right) d s^{\prime}\right]_{\vec{r}^{\prime} \in \partial \Omega_{1}}=\left[\frac{1}{r^{\prime}} \frac{\partial G}{\partial \theta^{\prime}}\left(r, \theta \mid r^{\prime}, \theta^{\prime}\right) u\left(r^{\prime}, \theta^{\prime}\right)\left(-d r^{\prime}\right)\right]_{\theta^{\prime}=\pi}} \\
& =-\frac{f\left(r^{\prime}\right) d r^{\prime}}{r^{\prime}}\left[\frac{\partial G}{\partial \theta^{\prime}}\left(r, \theta \mid r^{\prime}, \theta^{\prime}\right)\right]_{\theta^{\prime}=\pi}=-\frac{f\left(r^{\prime}\right) d r^{\prime}}{r^{\prime}} \frac{\partial}{\partial \theta^{\prime}}\left[-\ln r+\ln r^{*}-\ln r^{\dagger}+\ln r^{* \dagger}\right]_{\theta^{\prime}=\pi} \\
& =\frac{f\left(r^{\prime}\right) d r^{\prime}}{r^{\prime}} \frac{\partial}{\partial \theta^{\prime}}[\underbrace{\frac{1}{r} \frac{\partial r}{\partial \theta^{\prime}}-\frac{1}{r^{*}} \frac{\partial r^{*}}{\partial \theta^{\prime}}}+\frac{1}{r^{\dagger} \frac{\partial r^{\dagger}}{\partial \theta^{\prime}}-\frac{1}{r^{* \dagger}} \frac{\partial r^{* \dagger}}{\partial \theta^{\prime}}}]_{\theta^{\prime}=\pi} \\
& =\frac{f\left(r^{\prime}\right) d r^{\prime}}{r^{\prime}}[\underbrace{\frac{2 r^{\prime} r \operatorname{sen} \theta}{r^{\prime 2}+r^{2}+2 r^{\prime} r \cos \theta}}+\frac{\underbrace{\left(r^{\prime} r / b\right)^{2}+b^{2}+2 r^{\prime} r \cos \theta}}{]}
\end{aligned}
$$

cuja substituição em (21) fornece exatamente a equação (16), obtida por transformada de Fourier:

$$
\begin{aligned}
u_{2}(r, \theta) & =-\frac{1}{2 \pi} \int_{b}^{0} \frac{f\left(r^{\prime}\right) d r^{\prime}}{r^{\prime}}\left[\frac{2 r^{\prime} r \operatorname{sen} \theta}{r^{\prime 2}+r^{2}+2 r^{\prime} r \cos \theta}+\frac{2 r^{\prime} r \operatorname{sen} \theta}{\left(r^{\prime} r / b\right)^{2}+b^{2}+2 r^{\prime} r \cos \theta}\right] \\
& =\frac{r \operatorname{sen} \theta}{\pi} \int_{0}^{b}\left[\frac{1}{r^{\prime 2}+r^{2}+2 r^{\prime} r \cos \theta}+\frac{1}{\left(r^{\prime} r / b\right)^{2}+b^{2}+2 r^{\prime} r \cos \theta}\right] f\left(r^{\prime}\right) d r^{\prime} .
\end{aligned}
$$

\section{Conclusões}

É interessante constatar, num problema de domínio finito, o uso da transformada de Fourier. A possibilidade desse uso transparece com a mudança de variável em (9), mas também pode ser entendida resolvendo-se o problema da seção 2.2 pelo método alternativo, porém equivalente, de separar as variáveis. Assim se obtém um problema de autovalor de espectro contínuo na variável radial; portanto, para expandir a solução em termos das autofunções, é necessário realizar, não um somatório, mas uma integração, que é a inversa da própria transformada de Fourier usada.

Percebe-se a agradável possibilidade de não só se realizar o somatório infinito que surge na separação de variáveis [v. (5) e (7)] como também a de se efetuar a integral associada à transformada de Fourier inversa [v. (14) e (15)]. Sem isso não seria possível verificar tão facilmente que a solução obtida por esses dois métodos, a soma de (8) e (16), é igual à solução obtida pela função de Green determinada pelo método das imagens, a soma de (27) e (28); elas têm formas idênticas.

Corriqueiramente não ocorre essa agradável possibilidade (ela é excepcional), o que permite dizer que, comparativamente, o método da função de Green calculada pelo método das imagens leva mais diretamente a uma forma de solução mais aprimorada, mais fechada. Entretanto, este método tem aplicação bem menos vasta, sendo raro se conseguir determinar as imagens necessárias.

\section{Referências}

[1] Butkov, E. Física Matemática. LTC, Rio de Janeiro, 1988.

[2] Duffy, D. G. Green's Functions with Applications. Chapman \& Hall/CRC, Boca Raton, 2001.

[3] Gradshteyn, I. S. e Ryzhik, I. M., editado por Jeffrey, A. e Zwillinger, D. Table of Integrals, Series, and Products, Seventh Edition. Academic Press (Elsevier), Amsterdã, 2007.

[4] Zachmanoglou, E. C. e Thoe, D. W. Introduction to Partial Differential Equations with Applications. Dover, Nova York, 1976.

[5] Zill, D. G. e Cullen, M. R. Equações Diferenciais. Pearson/Makron Books, São Paulo, 2001. 